\title{
Distributions of Two Atoms Collisions over the Surface of the Condensed Phase
}

\author{
Sergey Zheltov ${ }^{1}$, Leonid Pletnev ${ }^{2}$ \\ ${ }^{1}$ Math Department, Tver State University, RU-170036, Tver, Russia \\ ${ }^{2}$ Dep of General scientific disciplines, Tver State Technical University, RU-170036, Tver, Russia Russia
}

\begin{abstract}
The processes of heat and mass transfer are closely related to the evaporation of a substance from the surface of the condensed phase. The interaction of outgoing molecules from the surface of the condensed phase with condensed phase molecules plays a fundamental role. A simpler case of evaporation is the departure of atoms from the surface of the condensed phase, i.e. the atoms overcome the potential barrier on the surface of the condensed phase. Depending on the evaporation rate, a Knudsen layer appears above the surface of the condensed phase. In this paper, based on the model of rigid spheres, the density distributions of the collision distances and the average values of the collision distances of two atoms emitted simultaneously from the surface of the condensed phase above the surface are analyzed. Distributions of the collision distance depending on the surface temperature, the size of the potential barrier, and the size of the evaporation area are obtained. Computer experiments were performed using the Monte Carlo method. To obtain the results of numerical simulation, a parallel algorithm adapted to calculations on graphics processors with CUDA technology was developed.
\end{abstract}

\section{Introduction}

The processes of heat and mass transfer in systems are related both to the interaction of gas with the walls of the systems [1-5] and to the interaction of the evaporating substance with the surface of the condensed phase [611]. It is of considerable interest to consider the evaporation process from a microscopic point of view. In this case, it is necessary to know the interaction potentials of molecules or atoms of a substance with each other or the walls of systems. Analysis of the evaporation of a substance consisting of molecules is a more complex task compared to the evaporation of a substance consisting of atoms, since when interacting with molecules, the exchange between the internal degrees of freedom of the molecules must be taken into account. With lowintensity evaporation of a substance, atoms or molecules can travel a sufficiently long distance before colliding [12-15], so there is a Knudsen layer near the wall surface and the surface of the condensed phase. In this layer, there are significant changes in the parameters that characterize the gas - temperature, density, etc. Due to the lack of accurate data on the interaction of atoms with each other, jumps of these parameters on the surface are introduced.

To describe the gas in this layer, the Boltzmann equation or model equations derived from it is used. The problems of describing a gas in this layer arise because the interaction processes are non-equilibrium, i.e. the velocity distribution function of gas atoms differs from the equilibrium distribution function. The development of computer technology has made it possible to use Monte Carlo and molecular dynamics methods to analyze the evaporation of a substance. It should be noted that even the use of modern computers and software does not allow for a detailed analysis of the evaporation process, since in real conditions it is necessary to calculate a huge number of outgoing molecules and, especially, their interaction with each other in the gas phase.

\section{Problem statement}

This paper presents the results of calculations of model computer experiments to study the distributions of collisions of two atoms over the surface of a condensed phase. The atoms flew simultaneously into the vacuum from a limited area of a square surface with a side $a=10^{-}$ ${ }^{9} \mathrm{~m}$. The model of atoms in the form of rigid spheres with a radius $R=1.5 \cdot 10^{-10} \mathrm{~m}$ was used. The masses of the atoms were assumed to be equal to $\mathrm{m}=40 \mathrm{AU}$. Fig. 1 shows a scheme of the departure of two atoms from the bounded surface of the condensed phase.

The coordinates of the atoms $\left(x_{\mathrm{i}} ; y_{\mathrm{i}}\right)$ flying out from the surface of the condensed phase and colliding over its surface were played out using a sensor of uniformly distributed random variables. The distance $a_{12}$ was defined as the distance between the centers of atoms and could be in the range $\left(3 \cdot 10^{-10} \mathrm{~m} ; 38.18 \cdot 10^{-10} \mathrm{~m}\right)$. The atomic velocity components $\left(v_{\mathrm{xi}} ; v_{\mathrm{yi}} ; v_{\mathrm{zi}}\right)$ were played out using a normal distribution sensor. If a collision between atoms occurred, the collision parameters were entered in the 
corresponding arrays; if not, a new pair of atoms was played.

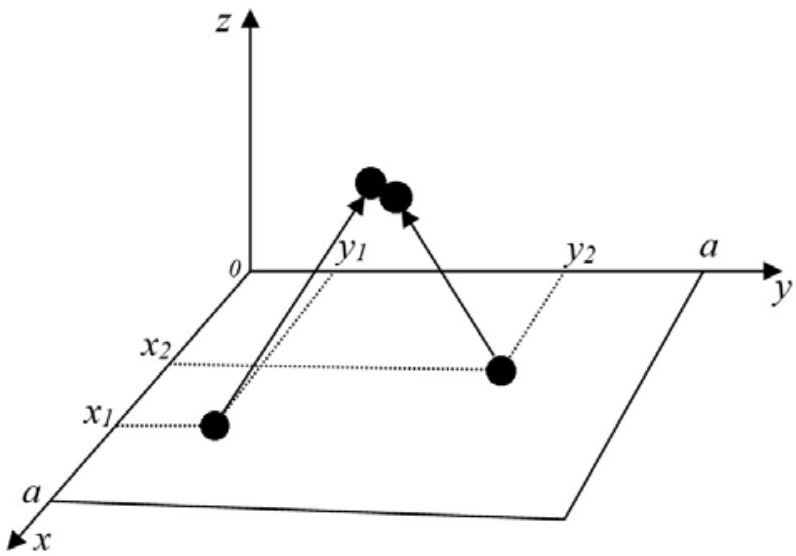

Fig. 1. Scheme of departure of two atoms from the surface of the condensed phase.

A special feature of the computer experiments is that the calculations are made taking into account the potential barrier on the surface of the condensed phase $U$ formed by the interatomic forces of the condensed phase. From the $z$ - component of each outgoing atom, a part of the energy equal to the value of the potential barrier $U$ is subtracted. If the kinetic energy of the $z$ component is greater than the value of the potential barrier $U$, then the atom was considered to have flown out. Otherwise, a new value of the $z$-component of the speed was played. It was found that the calculations depend on the dimensionless parameter $r=U / k T$, where $T$ is the surface temperature of the condensed phase and $k$ is the Boltzmann constant. As was found in [16,17], the average velocity component $v_{z}$ of the ejected atoms perpendicular to the surface of the condensed phase increases with an increase in the parameter $r$. The other two components of the velocities of the ejected atoms $v_{x}$ and $v_{y}$ did not change.

Computer experiments were performed using the Monte Carlo method for several hundred million pairs of atoms. The original problem has a so-called data parallelism and belongs to the SIMD class. Thus, it is possible to simulate the collision of several million pairs of atoms simultaneously. To increase efficiency, an algorithm that was adapted to computing on graphics processors with CUDA technology was developed. The calculations were performed on the NVIDIA Tesla K80 GPU of the heterogeneous HybriLIT platform, which is part of the multifunctional information and computing complex of the JINR information technology Laboratory (Dubna).

\section{Purpose of research}

Because not all ejected atoms collided with each other above the surface, is of interest to determine the density distributions of the distance $a_{12}$ between the colliding atoms at the time of departure on the surface of the condensed phase, i.e., surface distance for which collision over the surface is real. In addition to the densities of the $a_{12}$ distance distributions, the average distances of $a_{12}$ were obtained.

As a result of computer experiments, the density distributions of the distances of the collision of atoms on the surface of the condensed phase $z$ and the average distance values of collisions of atoms $Z_{\mathrm{av}}$ above the surface of the condensed phase were determined.

The densities of distributions of the total collision distances of $l$ atoms and the average values of the total collision distances of $l_{\mathrm{av}}$ atoms from the surface of the condensed phase were determined. In fact, this meant that the lengths of free paths of atoms before collisions were determined.

All the above distribution densities and average values are obtained for various values of the surface temperature of the condensed phase $T$, the value of the dimensionless parameter $r$, and the size of the square evaporation area with side $a$.

Additional studies were performed to obtain the densities of velocity distributions $v_{\mathrm{z}}$ between colliding atoms.

\section{Results of calculation}

The densities of distance distributions $a_{12}$ depending on the parameter $r$ are shown in Fig. 2. Normalization of all distribution densities was performed by dividing each value of the number in the cell by the largest value for all distributions. The distributions have maxima that depend on the value of the parameter $r$, which shift towards smaller distances $a_{12}$ as this parameter increases. This can be explained by the fact that as the parameter $r$ increases, the average values of the $v_{\mathrm{z}}$ components increase, and the atoms may collide if they are closer to each other. Computer experiments were performed for the surface temperature $T=50 \mathrm{~K}$. Calculations with other temperature values led to the same results. The temperature value changes the values of the average values of the components of the atomic velocities and, accordingly, the time before the collision, but not the point of collision of the atoms.

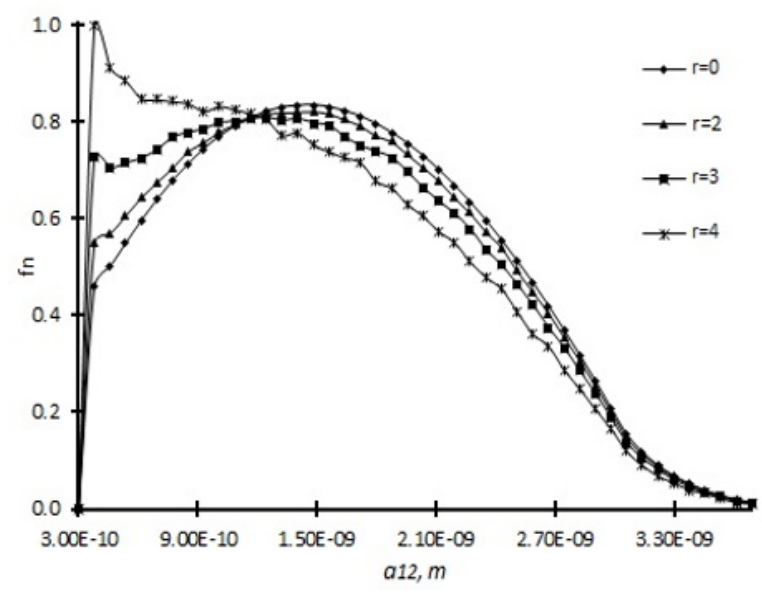

Fig. 2. Densities of distance distributions $a_{12}$.

It should be noted that the calculations were performed for the values of the parameter $r \in[0 ; 12]$. As it 
was found, the results of calculations change slightly with an increase in the parameter $r$ from 8 to 12 . The results of calculations obtained for the value $r=8$ can be considered as limiting results, i.e. atoms fly out from the surface according to the cosine law.

This is confirmed by the distribution of average distances $a_{12}$ depending on the parameter $r$, shown in Fig. 3 . The distribution of average distances $a_{12}$ does not depend on temperature and decreases with increasing parameter $r$.

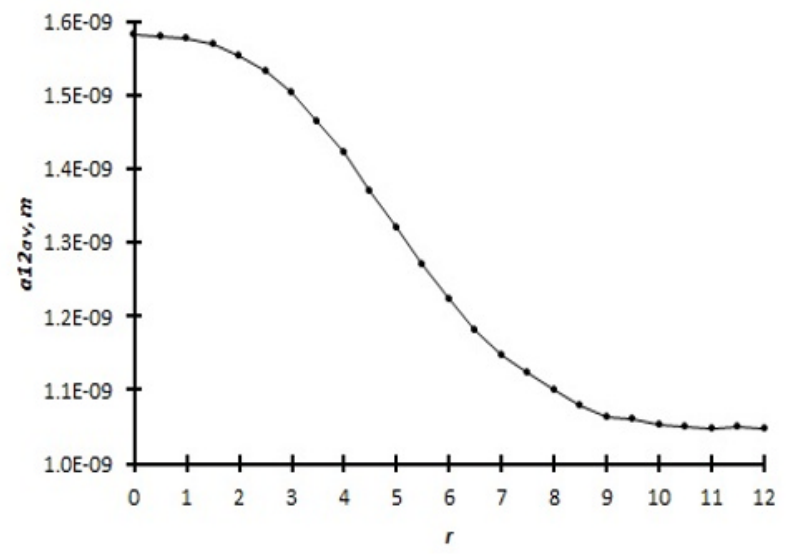

Fig. 3. Average distances $a_{12}$ between colliding atoms on the surface of a condensed phase.

As a result of calculations, the distances $z$ above the surface of the condensed phase at which the atoms collided above the surface of the condensed phase were determined. The value of $z$ was defined as the product of the atom with the minimum velocity for the time of movement before the collision. Fig. 4 shows the results of calculations of the density distributions of collision distances $z$ for several values of the parameter $r$. Calculations were performed for the surface of the condensed phase with a temperature $T=50 \mathrm{~K}$. As the parameter $r$ increases, the maxima of the distributions shift to the right, towards larger collision distances, and increase slightly. It is found that the distributions do not depend on the surface temperature, since the temperature affects the velocity components, i.e. the atoms collide faster, but at the same point.

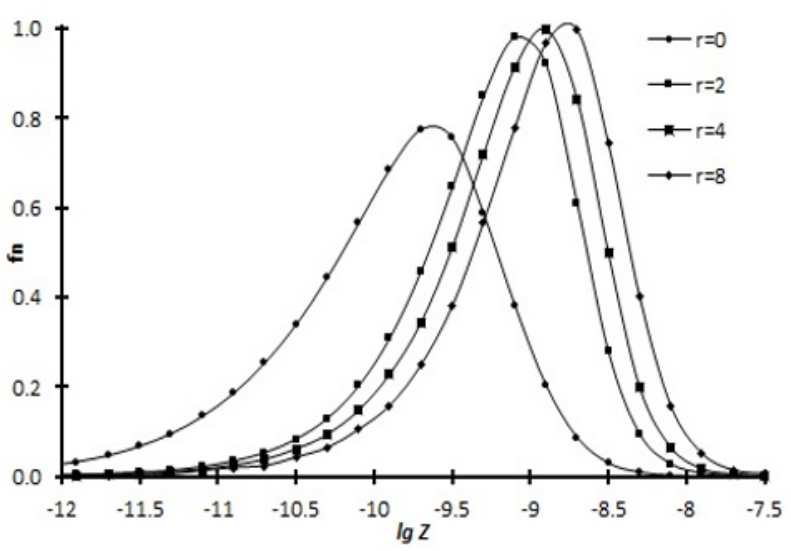

Fig. 4. Densities of distributions of collision distances $z$ depending on the parameter $r$.
Colliding atoms, after leaving the surface of the condensed phase, could fly out of the evaporation area. The free path of the atoms from the point of departure from the surface of the condensed phase to the point of collision of the atoms $l$ was determined. The value $l$ was defined as the square root of the sum of the squares of the components of the atomic runs along the axes of the coordinate system. This value, on average, is almost twice as large as the corresponding $z$ value. Fig. 5 shows the normalized densities of distributions of the distances of atomic runs to collisions $l$. It is found that for the value $l$, the regularities of the distribution densities are the same as for the distribution densities of the value $z$. As the parameter $r$ increases, the maxima of distributions increase and shift to the area of large $l$. Just like the collision distribution densities $z$ in Fig. 4, the distribution densities for $l$ do not depend on the surface temperature of the condensed phase.

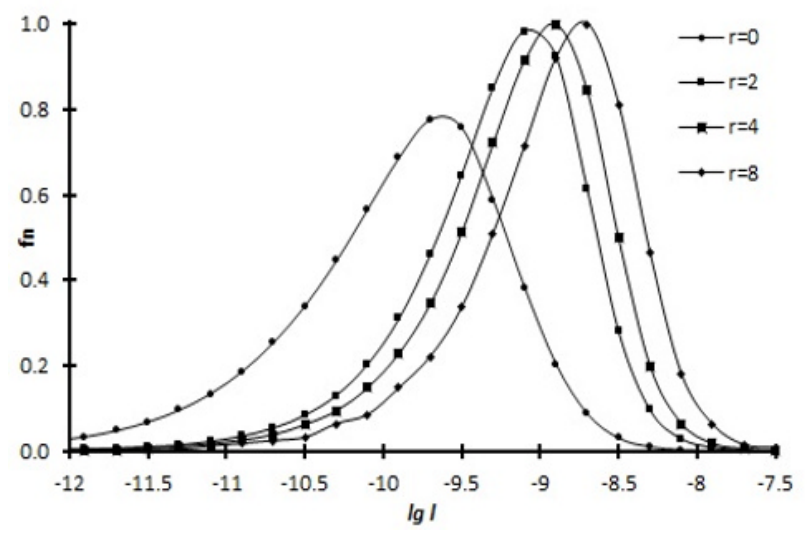

Fig. 5. Densities of distributions of collision distances $l$ depending on the parameter $r$.

Figure 6 shows the distributions of the average collision distances $z_{a v}$ and $l_{\mathrm{av}}$ depending on the parameter $r$. It is found that the distributions of the average values of these values do not depend on the surface temperature for the same reason as the distribution densities. As the parameter $r$ increases, the average distance $z_{a v}$ and $l_{\mathrm{av}}$ between colliding atoms increases.

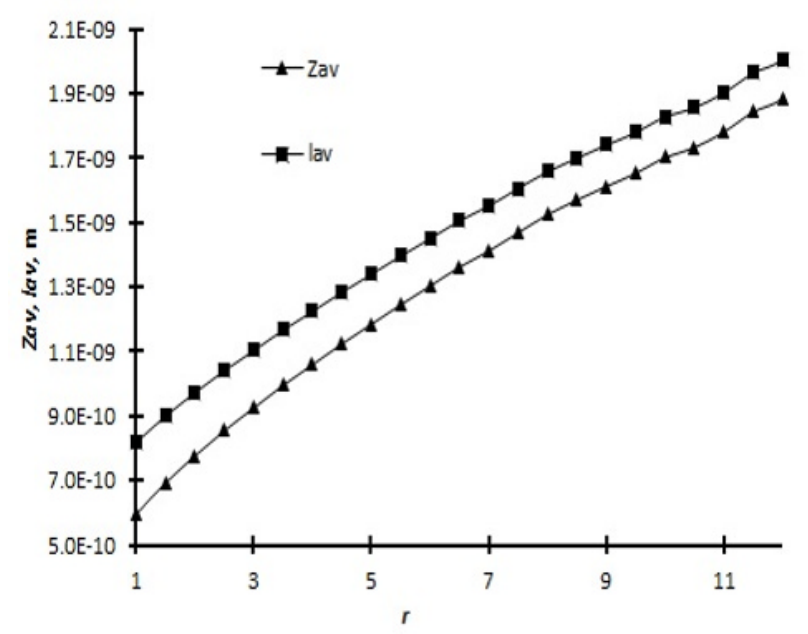

Fig. 6. Distributions of average $z_{a v}$ and $l_{\text {av }}$ values depending on the parameter $r$. 
A special series of computer experiments was performed to establish the dependences of the distribution densities and average collision values for the $z_{a v}$ and $l_{\mathrm{av}}$ values depending on the size of the evaporation area $a$. The results of calculations of distribution densities for the value $z$ for the parameter $r=2$ are shown in Fig. 7 . With the increase in the size of the evaporation area, the maxima decrease in height and shift to the right, towards larger values of $z$. It was found that the densities of $z$ collision distributions do not depend on the surface temperature.

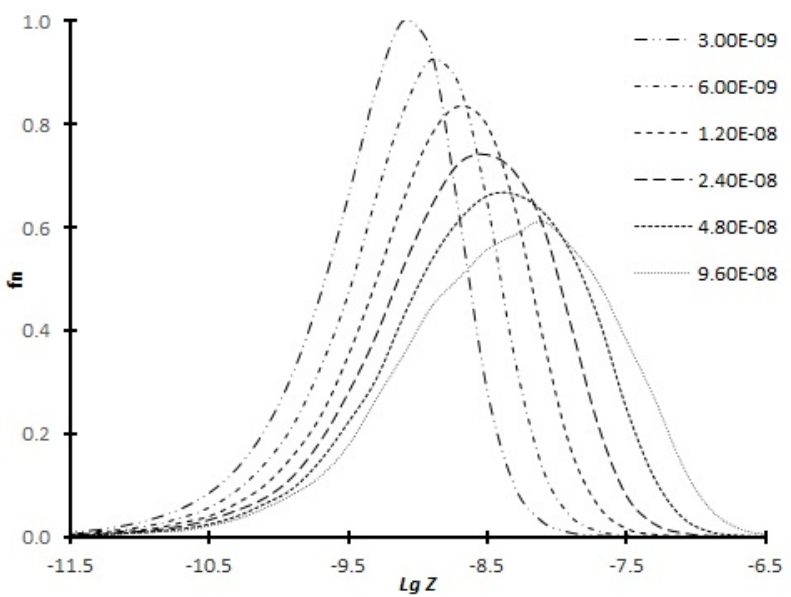

Fig. 7. Densities of distance distributions $z$ depending on the evaporation region $a . r=2$.

Fig. 8 shows the results of calculations of the distance distribution densities for the value $z$ for the parameter $r=8$. The obtained distributions do not depend on the surface temperature as well as for $r=2$. The forms of the $z$ distance distribution densities for $r=2$ and $r=8$ coincide, but are shifted to the left for $r=8$.

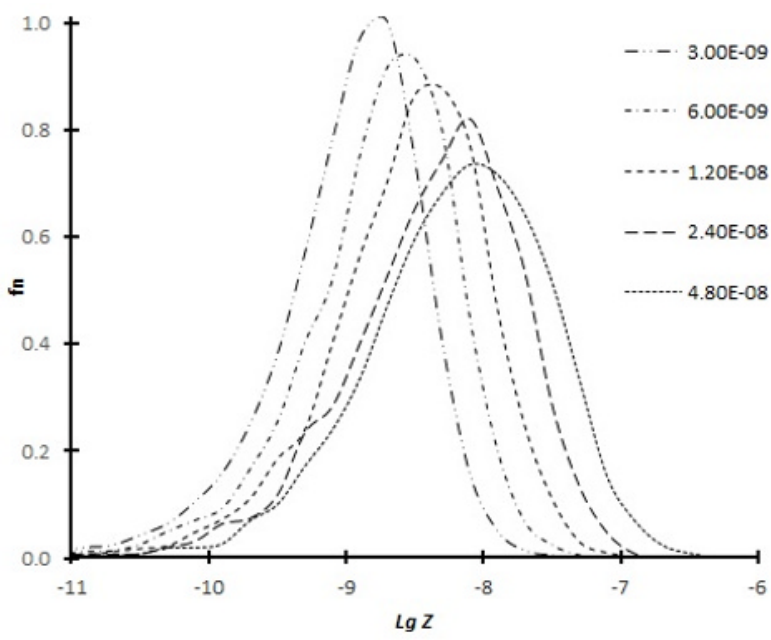

Fig. 8. Densities of distance distributions $z$ depending on the evaporation region $a . r=8$.

Densities of distributions of free path lengths $l$ were determined depending on the size of the evaporation area $a$ for temperatures $T=50 \mathrm{~K}$ and $T=100 \mathrm{~K}$. It is established that there is no difference between the densities of distributions of free path lengths $l$. The calculation results are shown in Fig. 9.
Similar results are obtained for the value of the parameter $r=8$. The densities of distance distributions $l$ do not depend on the surface temperature $T$. The calculation results are shown in Fig. 10.

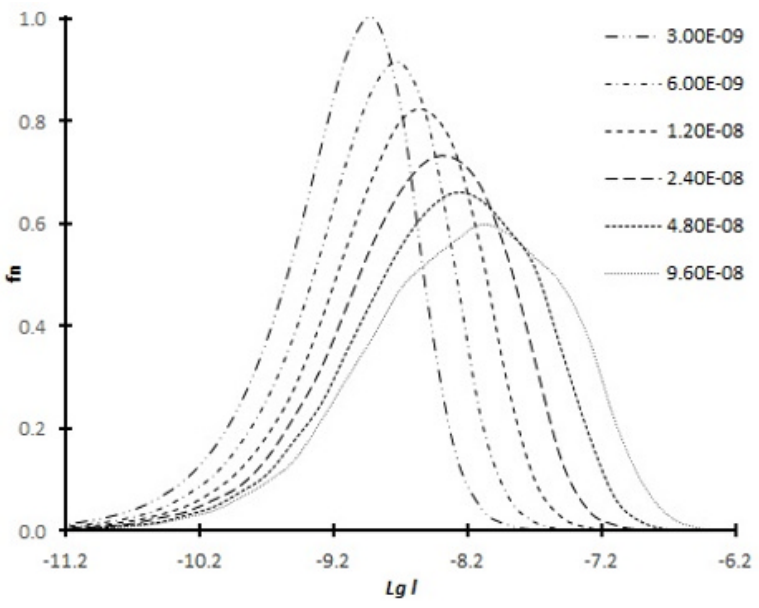

Fig. 9. Densities of distance distributions $l$ depending on the evaporation region $a . r=2$.

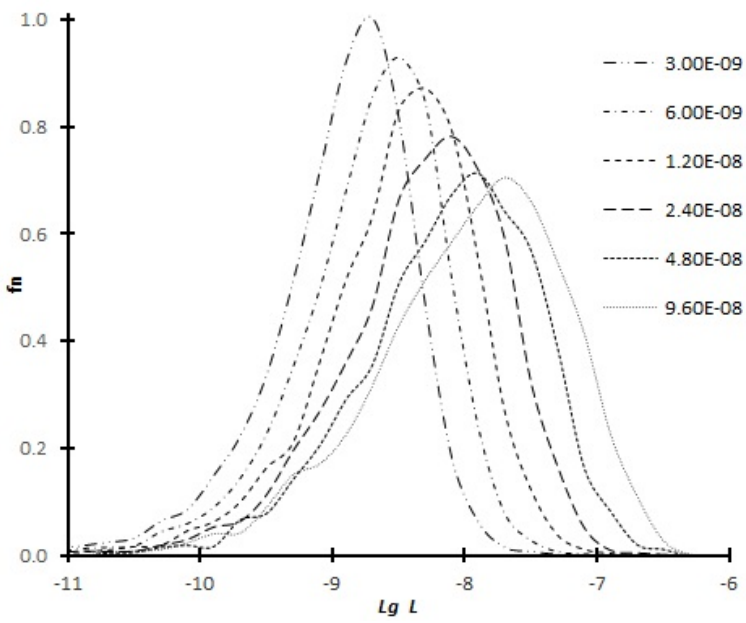

Fig. 10. Densities of distance distributions $l$ depending on the evaporation region $a . r=8$.

The distributions of the average distances $z_{a v}$ and $l_{\mathrm{av}}$ depending on the evaporation area $a$ are shown in Fig. 11. As the size of the evaporation region $a$ increases, the average values of these values change almost linearly.

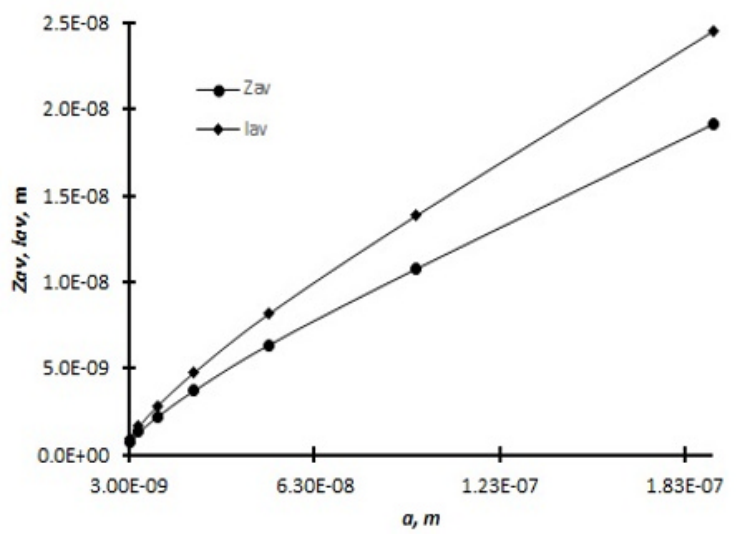

Fig. 11. Distributions of average distances $z_{a v}$ and $l_{\mathrm{av}}$ depending on the evaporation region $a . T=50 \mathrm{~K} . r=2$. 
The ratio between the corresponding $z_{a v}$ and $l_{\mathrm{av}}$ values remains constant. Similar distributions are obtained for other values of the surface temperature $T$ and the parameter $r$.

The distance between collisions of atoms depends on the velocity components of these atoms after overcoming the potential barrier on the surface of the condensed phase. The regularities of distributions of the average $v_{\mathrm{z}}$ components depending on the parameter $r$ for two temperature values are shown in Fig. 12.

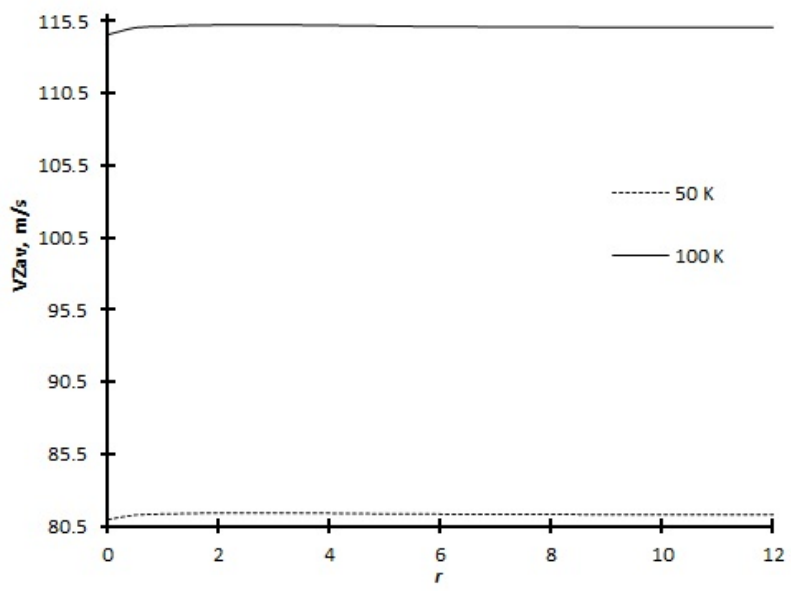

Fig. 12. Distributions of average values of atomic velocities $v_{\mathrm{z}}$ depending on the parameter $r . T=50 \mathrm{~K}$.

Distributions are almost parallel lines that do not depend on the parameter $r$. The ratio of the average velocities of the components $v_{\mathrm{z}}$ is equal to the square root of the inverse ratio of temperatures.

\section{Conclusions}

Computer experiments made it possible to determine the structure of the Knudsen layer in the framework of the proposed model of the collision of two atoms flying from a limited area of the condensed phase surface. Taking into account the influence of the interaction potential on the conditions of atomic collisions allowed us to obtain a more accurate structure of the Knudsen layer. The obtained densities of distributions of atomic collision distances allowed us to establish the complex structure of this layer.

Analysis of these distributions has shown that the main number of collisions of atoms occurs in a wide range of distances. From a few nanometers to hundreds of nanometers. To determine the Knudsen layer, you can use the average values of $z_{a v}$ and $l_{\mathrm{av}}$, which almost coincide with the maxima of the distributions.

The results of calculations for the collision of two atoms can be used as a basis for calculating the collisions of three atoms simultaneously flying from the surface.

\section{References}

1. J. Lee, T. Laoui, R. Karnik, Nat. Nanotech., 9 (2014)
2. Y. Li, M.A. Alibakhshi, Y. Zhao, C. Duan, Nano Lett., 17 (2017)

3. K.L. Wilke, B. Barabadi, Z. Lu, T. Zhang, E.N. Wang, Appl. Phys. Lett., 111 (2017)

4. R. Xiao, S.C. Maroo, E.N. Wang, Appl. Phys. Lett., $102(2013)$

5. A. Frezzotti, Phys. Fluids, 23 (2011)

6. A.H. Persad, C.A. Ward, Chem. Rev., 116 (2016)

7. M. Knudsen, Ann. Phys., 352 (1915)

8. F.G. Tcheremissine, Comput. Math. Phys., 46 (2006)

9. T. Ishiyama, S. Fujikawa, T. Kurz, W. Lauterborn, Phys Rev E, 88 (2013)

10. M. Kon, K. Kobayashi, M. Watanabe, Int. J. Heat Mass Transfer, 99 (2016)

11. K. Kobayashi, K. Sasaki, M. Kon, H. Fujii, M. Watanabe, Microfluid Nanofluid, 21 (2017)

12. S. Cheng, J.B. Lechman, S.J. Plimpton, G.S. Grest, J. Chem. Phys., 134 (2011)

13. M. Kon, K. Kobayashi, M. Watanabe, Phys. Fluids, 26 (2014)

14. M. Kon, K. Kobayashi, M. Watanabe, Eur. J. Mech. B Fluids, 64 (2017)

15. K. Kobayashi, K. Hori, M. Kon, K. Sasaki, M. Watanabe, Heat Mass Transfer, 52 (2016)

16. L.V. Pletnev, N.I. Gamayunov, V.M. Zamyatin, Mathematical Models of Non-Linear Excitations, Transfer, Dynamics, and Control in Condensed Systems and Other Media. L.A. Uvarova (eds) (Springer, Boston, MA. 1999) https://doi.org/10.1007/978-1-4615-4799-0_13

17. L.V. Pletnev, J. Monte Carlo Methods and Applications, 6, 3 (2000) 